\title{
Unusual Cause of Symptomatic Bradycardia. Passing Out Once Looking Right!
}

\begin{abstract}
A 63 year old white man with hypertension presents complaining of episodes of light headedness and generalized weakness, in the last year, more frequent in the last few weeks. He has noticed an overall decline in heart rate (HR) while exercising on treadmill. Atenolol dose was decreased a month ago with no improvement. Physical exam revealed a right sided goiter, otherwise unremarkable. Labs, EKG, Stress test and Echocardiogram were within normal limits. No pauses on the monitor. Carotid massage revealed a significant decrease in HR from 78 to 45 with lightheadedness when done on the right side. Atenolol was discontinued and he was discharged with event monitor. He returned to the hospital few days later with recurrent symptoms. Event monitor showed a sudden decrease in HR from 120 to 70 during exertion. Further questioning showed that all episodes were precipitated by turning his head to the right side. We suspected that the neck mass is compressing the carotid sinus and causing bradycardia. CT neck showed a large goiter compressing on adjacent structures. Thyroidectomy was done and patient was seen six weeks later with no more spells.
\end{abstract}

Keywords: Symptomatic bradycardia; Reversible bradycardia; Chronotropic incompetence; Lightheadedness

Case Report
Volume 2 Issue 4 - 2015
Abdrhman Hamo*, Courtney Staton and
Esam Baryun
Department of Cardiovascular Medicine, Marshall University,
USA
*Corresponding author: Abdrhman Hamo, Department
of Cardiovascular Services and Internal Medicine, Marshall
University, Joan C. Edwards School of Medicine, 1249 15th
Street, Suite 4000, Huntington, West Virginia, USA, Tel: 304-
633-5052; Fax: 304-691-8530; Email: drarh77@yahoo.com;
hamo@marshall.edu

Received: March 30, 2015 | Published: April 15, 2015
Abbreviations: ECG: Electrocardiogram; CT: Computed Tomography; HR: Heart Rate

\section{Introduction}

Reversible causes of bradycardia include drug-induced bradycardia; increased vagal tone; increased intra cranial pressure, carotid sinus pressure, hypothyroidism, hypoxia , and obstructive sleep apnea. It is very important to rule out completely reversible cases of bradycardia before a permanent pacemaker is indicated. We are presenting a very rare case of symptomatic bradycardia and episodes of chronotropic incompetence secondary to a goiter compressing the carotid sinus which was treated successfully.

\section{Case Report}

A 63-year old hypertensive gentleman presented with episodes of light headedness, chest tightness, and generalized weakness, in the last year which became more frequent in the last few weeks. Patient recently noticed that his heart rate (HR) is getting obviously slower while he exercises on the treadmill. $\mathrm{He}$ is on Atenolol $25 \mathrm{mg}$ a day for hypertension and the dose was decreased to $12.5 \mathrm{mg}$ a month ago without benefit. On physical examination he has a right sided asymmetric goiter, otherwise unremarkable. The EKG showed sinus bradycardia with HR of 55 bpm (Figure1). Laboratory analyses revealed intact thyroid function tests, cardiac enzymes, and electrolytes. Carotid massage was negative for hypersensitivity syndrome (no pause or hypotension). A treadmill stress test and 2D-Echo were unremarkable. Atenolol was discontinued and he was discharged home with an event monitor. Unfortunately a few days later the patient had recurrent symptoms. The event monitor showed no evidence of bradycardia but at the time of symptoms he had a sudden decrease in his HR from 110 to 70 (Figure 2), which was inappropriate, as that happened while walking upstairs .

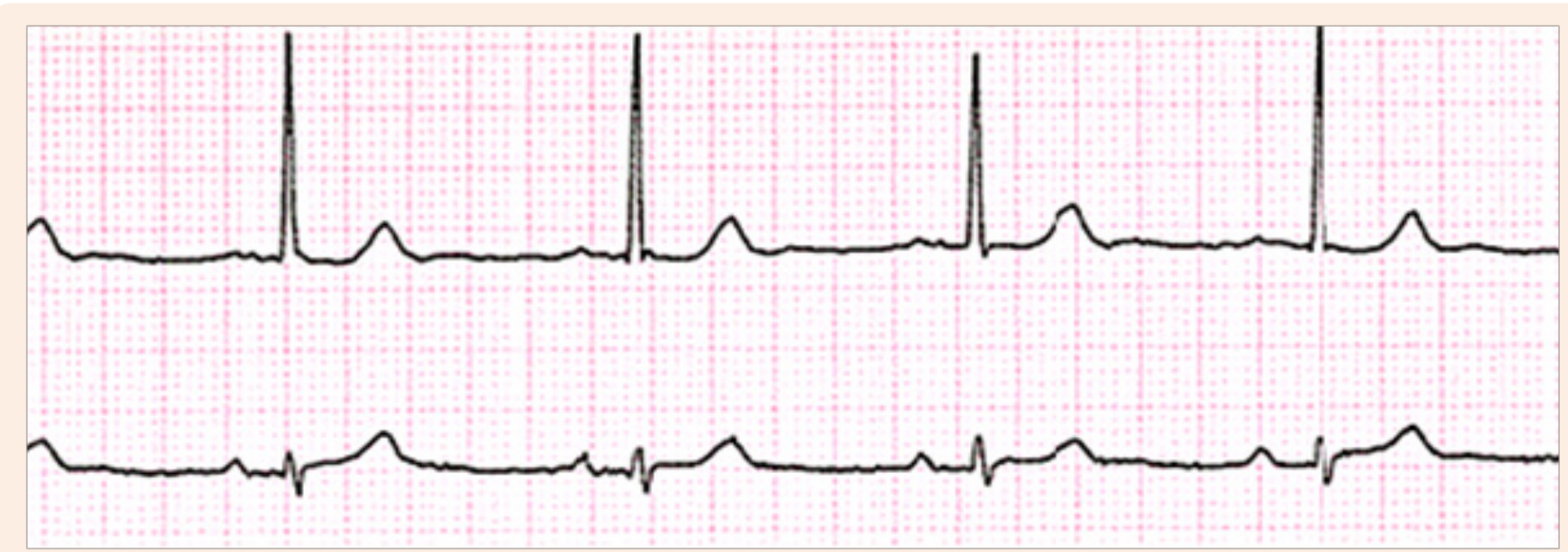

Figure 1: The EKG showed sinus bradycardia with HR of $55 \mathrm{bpm}$. 


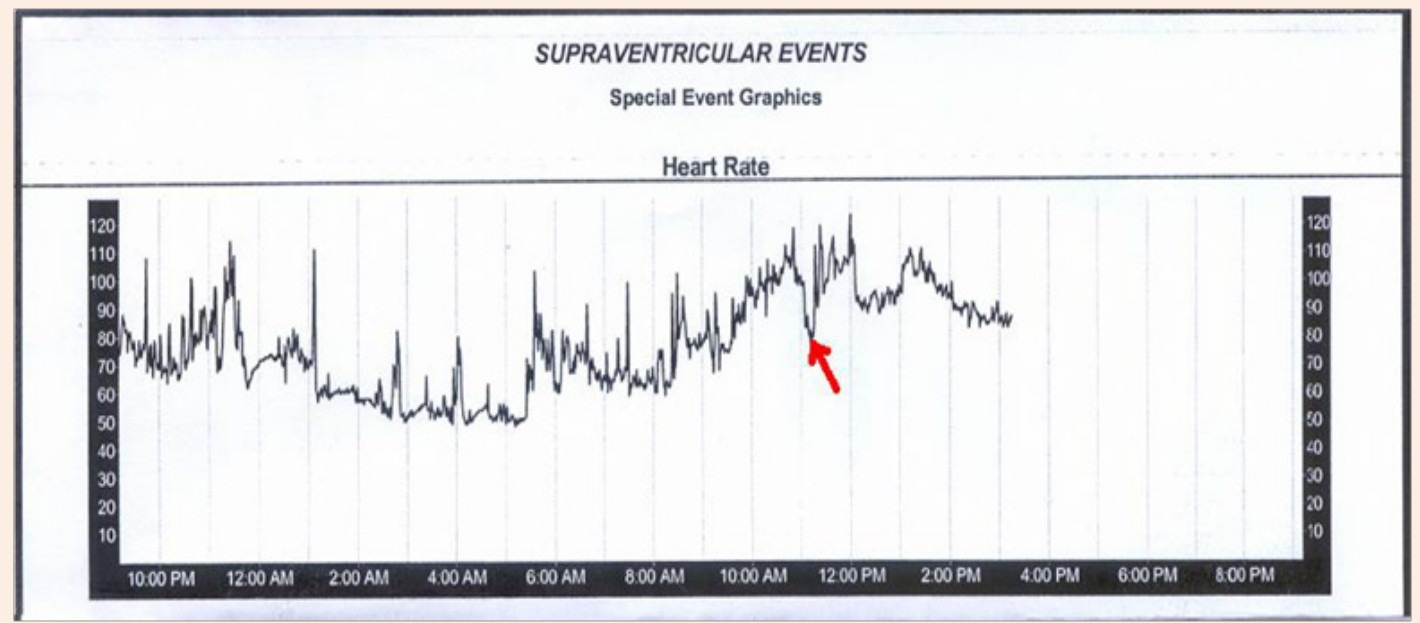

Figure 2: Sudden drop in heart rate during exertion was inappropriate during exertion and caused symptoms.

Upon further questioning it was found that the patient's symptoms were always precipitated by turning his head to the right side...!! "When he was on vacation the spells occurred while he was looking out the window to the right; whereas at home after running up stairs there was a sharp right turn." Therefore there was a high suspicion that a neck mass is compressing the carotid sinus causing a reflex bradycardia. Consequently a CT neck was performed that revealed a large goiter compressing on adjacent structures including the right carotid sinus (Figure 3 \& 4).

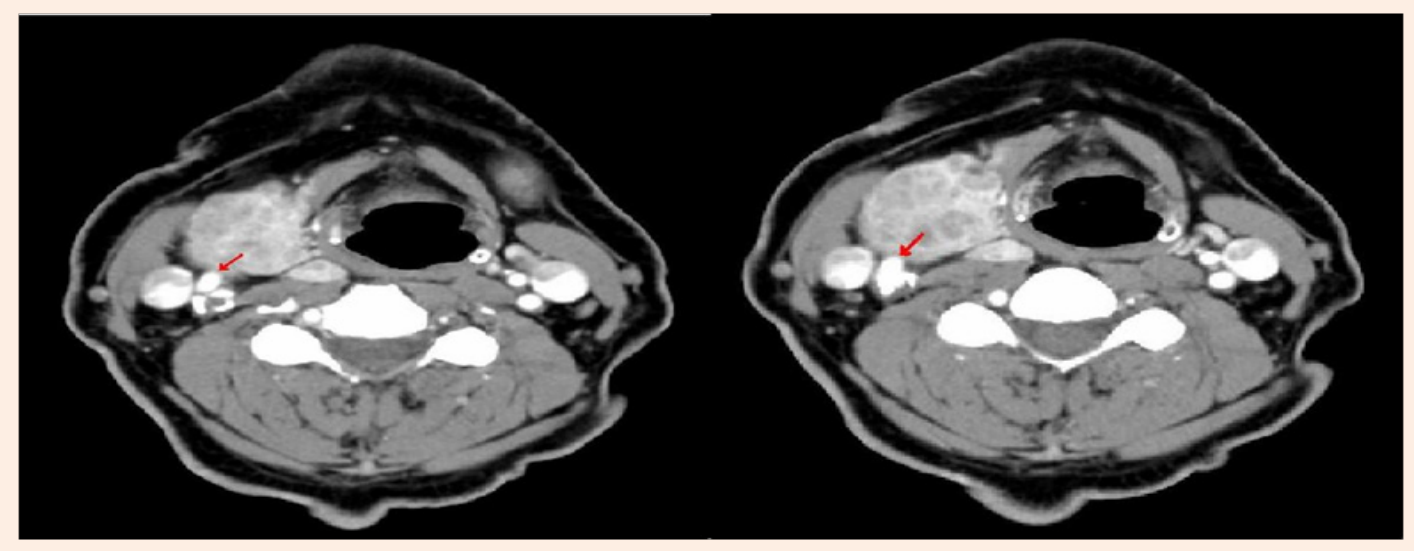

Figure 3: Compression on the right carotid sinus (Right image) and on carotid artery bifurctation (Left image).

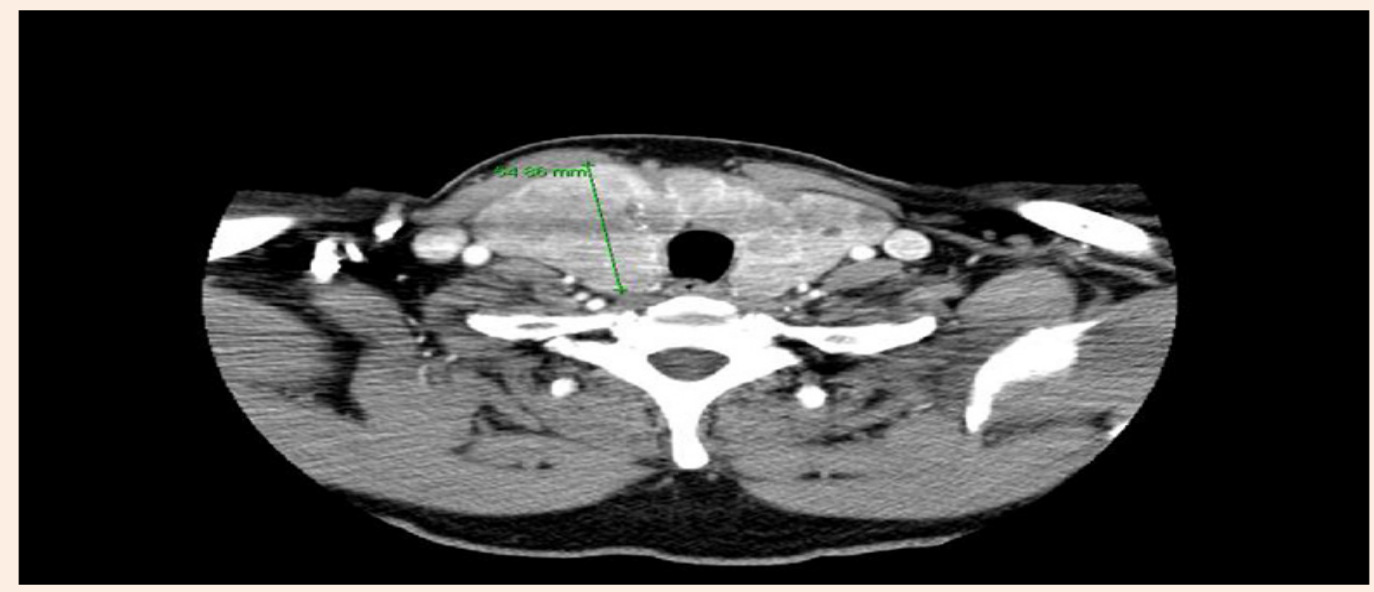

Figure 4: Asymmetric large goiter confirmed by CT scan of the neck. 
Thyroidectomy was performed with a preoperative temporary trans-venous pacemaker placement to prevent profound bradycardia during surgery, and the patient was seen six weeks later with no more spells of weakness or lightheadedness. In a patient who presents with bradycardia or syncope of unknown origin, a thorough history and physical examination can sometimes provide clues suggesting unusual causes of bradycardia like our case. The most common reversible causes of bradycardia include drug-induced bradycardia [such as beta-blockers, calciumchannel blockers, and antiarrhythmic agents]; increased vagal tone; and electrolyte abnormalities. Other reversible causes include: Increased Intra Cranial Pressure, Carotid sinus pressure, Hypothyroidism, Hypoxia , and Obstructive Sleep Apnea.

Management of bradycardia depends on multiple factors, such as the acuity and severity on presentation, the nature and reversibility of precipitating events, and underlying electrophysiological and cardiac abnormalities. Patients presenting with haemodynamic compromise, signs of cerebral hypoperfusion, progressive heart failure, or life-threatening arrhythmias secondary to bradycardia require urgent treatment. The role of medicines in managing bradycardia is generally limited to emergency situations. Search for a medication that may be depressing sinus node function. As an example, a beta blocker, non dihydropyridine calcium channel blocker, or sympathetic blocker can be replaced in hypertensive patients with drugs that do not affect SA node function, such as an angiotensin converting enzyme inhibitor. Sympatholytic agents should not be discontinued abruptly, since a withdrawal syndrome may be seen characterized by severe hypertension and, with abrupt beta blocker withdrawal, the precipitation of angina.

Pharmacologic therapy may be important in an acute myocardial infarction when the SA node is depressed by excessive parasympathomimetic activity or possibly ischemia. In this setting, the SA node rate generally increases after the administration of a vagolytic drug, such as atropine [1]. Longterm pharmacologic therapy is largely ineffective in chronic sinus bradycardia. Symptomatic bradycardia is a Class I indication for permanent pacemaker implantation [2]. Permanent pacemaker is the only reliable therapy for symptomatic bradycardia in the absence of extrinsic and reversible etiologies such as increased vagal tone, hypoxia, hypothermia, and drugs. We are generally in agreement with the 2008 American College of Cardiology/ American Heart Association/Heart Rhythm Society (ACC/AHA/ HRS) device guidelines [2,3].

\section{Conclusion}

It is very important to rule out completely reversible causes of bradycardia before a permanent pacemaker is indicated. This is a very rare case of symptomatic bradycardia and episodes of chronotropic incompetence secondary to a goiter compressing the carotid sinus which was treated successfully.

\section{References}

1. Zimetbaum PJ, Josephson ME (2003) Use of the electrocardiogram in acute myocardial infarction. N Engl J Med 348(10): 933-940.

2. Epstein AE, DiMarco JP, Ellenbogen KA, Estes NA, Freedman RA, et al. (2008) ACC/AHA/HRS 2008 Guidelines for Device-Based Therapy of Cardiac Rhythm Abnormalities: a report of the American College of Cardiology/American Heart Association Task Force on Practice Guidelines (Writing Committee to Revise the ACC/AHA/NASPE 2002 Guideline Update for Implantation of Cardiac Pacemakers and Antiarrhythmia Devices): developed in collaboration with the American Association for Thoracic Surgery and Society of Thoracic Surgeons. Circulation 117: e350.

3. Tracy CM, Epstein AE, Darbar D, DiMarco JP, Dunbar SB, et al. (2012) ACCF/AHA/HRS focused update of the 2008 guidelines for device-based therapy of cardiac rhythm abnormalities: a report of the American College of Cardiology Foundation/American Heart Association Task Force on Practice Guidelines and the Heart Rhythm Society. [corrected]. Circulation 126(14): 1784-1800. 